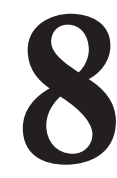

\title{
LA CESIÓN DE RESPONSABILIDAD EN LA EVALUACIÓN: UNA ESTRATEGIA ADAPTADA AL ESPACIO EUROPEO DE EDUCACIÓN SUPERIOR
}

\author{
(ASSIGNMENT OF RESPONSIBILITY IN EVALUATION: \\ A STRATEGY ADAPTED TO THE EUROPEAN HIGHER \\ EDUCATION AREA)
}

Juan Antonio Moreno-Murcia, Adolfo Aracil y Raúl Reina Universidad Miguel Hernández de Elche

DOI: 10.5944/educxx1.17.1.10710

\section{Cómo referenciar este artículo/How to reference this article:}

Moreno Murcia, J.A.; Aracil, A, y Reina, R. (2014). La cesión de responsabilidad en la evaluación: una estrategia adaptada al Espacio Europeo de Educación Superior. Educación XX1, 17 (1),183200. doi: 10.5944/educxx1.17.1.10710.

Moreno Murcia, J.A.; Aracil, A, y Reina, R. (2014). Assignment of responsibility in evaluation: a strategy adapted to the European Higher Education Area. Educación XX1, 17 (1),

183-200. doi: 10.5944/educxx1.17.1.10710.

\section{RESUMEN}

Este estudio prospectivo se centró en conocer la influencia que la cesión a los estudiantes de la responsabilidad sobre la evaluación tenía en la percepción de su propia autonomía, su motivación intrínseca, así como en la creencia del alumnado sobre el valor y el rol que debería tener la evaluación. 98 estudiantes universitarios de cuarto curso de la Licenciatura en Ciencias de la Actividad Física y del Deporte respondieron cuestionarios validados sobre el entrenamiento a favor de la autonomía, la autonomía percibida, la motivación intrínseca y la responsabilidad del alumnado en la evaluación. Y preguntas abiertas y cerradas relativas a la participación del alumnado en la evaluación en grupo, antes y después de una intervención didáctica de ocho meses de duración, basada en la cesión al alumnado de la responsabilidad en la evaluación. Tras un análisis descriptivo, de correlación y prueba t para muestras relacionadas, los resultados mostraron que este tipo de intervención incrementó la percepción de apoyo a la autonomía, la motivación intrínseca y el valor y rol concedido a la cesión de responsabilidad en la evaluación. Los participantes describieron este programa como un buen sistema de enseñanza-aprendizaje, novedoso y motivador. Estos resultados permiten apoyar el uso de programas de cesión de responsabilidad en la evaluación en la educación superior. 


\section{ABSTRACT}

In this study we undertake the prospective study of the impact of assignment of responsibility on evaluation to the students on their perception on its own autonomy, intrinsic motivation and their beliefs on the evaluation process. 98 undergraduate students at their fourth academic year of the Sports Sciences Degree participated in this study. Before and after a didactical intervention of 8 months of duration -in which responsibility on evaluation was partly assigned to the students-, participants responded a series of validated questionnaires on autonomy supportive coaching, autonomy self-perception, intrinsic motivation and on the role of students in the evaluation process, as well as several open questions on their beliefs on the participation of students in the evaluation process. Statistical differences were found between both time-points on the self perception of autonomy supportive coaching, intrinsic motivation and the role that assignment of responsibility should have in higher education. Participants described the intervention as a good learning strategy, new and motivating. These results support the use of strategies of assignment of responsibility on evaluation at higher education degrees.

\section{INTRODUCCIÓN}

En la esfera académica del estudiante, el concepto de «evaluación» suele estar fuertemente asociado al de «calificación», y ambos están estrechamente relacionados tanto con los productos de aprendizaje como con la satisfacción con los mismos (Özgüngör, 2009). Sin embargo, ambos hacen referencia a dos procesos diferenciados dentro de la enseñanza. Mientras la evaluación intenta establecer los criterios sobre los que juzgar la calidad del proceso de enseñanza-aprendizaje, la calificación se refiere a una parte incluida en la evaluación, que viene exigida administrativamente por el hecho de tener que colocar el profesorado, al final del periodo de enseñanza-aprendizaje, una puntuación a cada estudiante. Estamos, en ambos casos, ante una decisión compleja que suele ser una fuente constante de preocupaciones y dudas, tanto del discente como del docente. Por ello, encontrar una forma coherente de integrar la evaluación y la calificación (en lo que a veces representa finalidades opuestas), es una necesidad real del sistema educativo, aspecto éste que el Espacio Europeo de Educación Superior (EEES) contempla como uno de sus cambios. En el proceso de convergencia al EEES el cambio fundamental de la universidad europea lo va a ser en el aspecto conceptual: una nueva Universidad basada en la formación continuada de la persona que aprende a lo largo de toda su vida. Por ello, el trabajo académico deberá estar centrado en el estudiante, que pasa a ocupar un papel activo dentro del proceso educativo (Aracil, 2010), adoptando el docente un rol de «gestor» del proceso de aprendizaje del estudiante, garante de la calidad y significatividad del mismo. Más aún, en la evaluación de los aprendizajes de 
los universitarios se apuesta por permitir a los estudiantes a participar en su propia evaluación (Mateo y Martínez, 2005). Esta «nueva Universidad europea» deberá, pues, ajustar su organización, y sus estrategias metodológicas, incluyendo la evaluación, al nuevo marco en el que se encuadra.

La implementación de acciones educativas que conllevan un trabajo colaborativo, generalmente mediante proyectos o trabajo en grupo, ha sido una de las principales consecuencias de esta nueva realidad educativa (Elliott y Higgins, 2005). El aprendizaje colaborativo ha sido reportado como un medio de contrastada eficacia para la práctica profesional (Callaghan, Watts, McCullough, Moreau, Little, Gamroth y Durnford, 2009), aspecto éste fundamental en la educación superior. Sin embargo, determinadas variables interpersonales condicionan sobremanera dicho proceso, de manera que el modo en que se gestionen la responsabilidad otorgada en la calificación, puede determinar el éxito de la actividad formativa (van Genni, Segers y Tillema, 2009). Nos referimos a aspectos como la seguridad de la persona, la tolerancia a la diversidad de nivel entre miembros, el nivel de interdependencia entre los componentes del grupo, o el esfuerzo (van Genni, Segers y Tillema, 2010). Uno de los aspectos que más podría influir aquí es una falta de actitud positiva de alguno de los componentes para con el trabajo o bien del grupo. De acuerdo con Freeman y McKenzie (2002), los estudiantes no ven justo la valoración del trabajo realizado, si las aportaciones de los diferentes componentes no ha sido equitativa, pudiendo incluso a conllevar sentimientos de insatisfacción con el trabajo realizado (Toynbee-Wilson, 2001), sobre todo en aquellos compañeros que hubieren tenido un menor nivel de implicación.

El nivel de implicación, tanto de cada participante como de la propia dinámica grupal, condicionará el desarrollo y resultado del proceso de enseñanza-aprendizaje. Así, la determinación de la responsabilidad a través del compromiso del estudiante resulta a veces preocupante para el docente cuando al alumnado se le cede responsabilidades, de manera que los mecanismos para responsabilizarlos en la evaluación hacen referencia al interés en la organización e implicación de las tareas de aprendizaje (Moreno, Vera y Cervelló, 2009), por lo que resulta interesante inculcar en los estudiantes la capacidad de reflexionar críticamente sobre sus propias acciones y su aprendizaje (Del Valle, Ruiz, Vega, Velázquez y Díaz, 2005).

Así pues, el objetivo del presente estudio fue comprobar el efecto que un programa de trabajo basado en cesión de responsabilidad en la evaluación, a través del apoyo a la autonomía, tuvo en la creencia del propio alumnado sobre la misma, así como en la mejora de la percepción de autonomía, la motivación intrínseca, y el valor y rol de la evaluación. La hipótesis de trabajo se fundamenta en el modelo jerárquico de motivación intrínseca y extrinseca de Vallerand (Vallerand, 1997), que incluye entre sus elementos 
conceptuales la presencia de unos mediadores entre los desencadenantes y sus consecuencias. Se partió de la hipótesis de que un programa de cesión al estudiante universitario de la responsabilidad en la evaluación (como desencadenante) conseguiría aumentar su percepción de autonomía, y a través de ella (como mediador), su motivación intrínseca y el valor y rol que el alumnado atribuye a la evaluación (como consecuencias).

\section{MÉTODO}

\section{Participantes}

La muestra estuvo compuesta por 98 estudiantes ( 77 varones y 21 mujeres) universitarios de cuarto curso de la titulación de Ciencias de la Actividad Física y del Deporte de la Universidad Miguel Hernández de Elche, del curso académico 2008-09, con una edad media de 22.76 años $(D T=3.15)$.

\section{Instrumentos}

Cuestionario de percepción de soporte de la autonomía en el proceso de entrenamiento (ASCQ). Se empleó la traducción al castellano (Conde, SáenzLópez, Carmona, González-Cutre, Martínez Galindo, y Moreno, 2010) del Autonomy-Supportive Coaching Questionnaire de Conroy y Coatsworth (2007). Este cuestionario está compuesto por un total de nueve ítems agrupados en dos dimensiones: cinco ítems que miden el interés en la opinión del practicante (e.g. «Mi docente me permite elegir sobre lo que hacemos en clase») y cuatro ítems que evalúan la valoración del comportamiento autónomo (e.g. «Mi docente me valora por las cosas que elijo hacer en clase»). El cuestionario está encabezado por la frase «En mis prácticas...», respondiéndose mediante una escala de respuesta tipo Likert de 1 (totalmente en desacuerdo) a 7 (totalmente de acuerdo). Los alfas de Cronbach obtenidos fueron para la dimensión interés $\alpha$ pre $=.84 \mathrm{y} \alpha$ post $=.93$, y para la dimensión valoración $\alpha$ pre $=.81 \mathrm{y} \alpha$ post $=.91$.

Autonomía. Se utilizó el factor autonomía de la Escala de Mediadores Motivaciones en el Deporte (EMMD) de González-Cutre Coll, Martínez Galindo, Alonso, Cervelló, Conte y Moreno (2007). Estaba compuesta por ocho ítems (e.g. «Mi docente me valora por las decisiones que tomo»), precedidas de la frase «En mis clases...» y las respuestas fueron recogidas en una escala tipo Likert, donde 1 correspondía a totalmente en desacuerdo y 5 a totalmente de acuerdo. La consistencia interna obtenida fue de $\alpha$ pre $=.85 \mathrm{y} \alpha$ post $=.93$.

Motivación intrínseca. Se empleó el factor motivación intrínseca de la Escala del Locus Percibido de Causalidad (PLOC Scale) de Goudas, Biddle y 
Fox (1994), validada al contexto español por Moreno, González-Cutre y Chillón (2009). Este factor, encabezado por el enunciado «Participo en esta clase de educación física...», está compuesto por cuatro ítems (e.g. «Porque disfruto aprendiendo nuevas habilidades») que se responden con una escala tipo Likert que va de 1 (totalmente en desacuerdo) a 7 (totalmente de acuerdo). La consistencia interna reveló un valor $\alpha$ de .89 para la toma pre y .93 para la toma post.

Escala de Responsabilidad del alumnado en la Evaluación en Educación Física (ERAEEF). La escala de Moreno, Vera y Cervelló (2006) pretende conocer la importancia que el alumnado le concede a la cesión de responsabilidad en la evaluación. Está compuesta de dos factores: el primero, formado por cinco ítems, hace referencia al valor que el alumnado le da a la cesión de responsabilidad que el docente le otorga para su participación en el resultado de la evaluación (e.g. «Los estudiantes deben interesarse por decidir la nota que se merecen en la asignatura»). El segundo factor, formado por seis ítems, se refiere al rol que juega el alumnado en la cesión de responsabilidad en el proceso de la evaluación (e.g. «En mi clase los estudiantes le decimos al docente la opinión que tenemos de nuestra habilidad físico-deportiva»), valora el grado de participación que el alumnado tiene en la evaluación de la asignatura, determinado por la percepción del papel participativo que tienen de su evaluación y la de sus compañeros. Las respuestas a dicho cuestionario son cerradas y responden a una escala tipo Likert cuyos rangos de puntuación oscilaban entre 0 , valor que corresponde a totalmente en desacuerdo, y 10, valor correspondiente a totalmente de acuerdo con lo que se le planteaba. Los alfas de Cronbach obtenidos fueron para la dimensión valor en la cesión $\alpha$ pre $=.83$ $\mathrm{y} \alpha$ post $=.86$, y para la dimensión rol en la cesión $\alpha$ pre $=.81 \mathrm{y} \alpha$ post $=.84$.

Preguntas sobre la participación en la evaluación. Estuvo compuesta por dos preguntas cerradas (¿En qué medida consideras que tu intervención en el diseño, exposición y defensa del proyecto contribuirá a la calificación final de tu grupo?, valorada en una escala Likert cuyos rangos de puntuación oscilaban entre 1 , valor que corresponde a nada, y 5 , valor correspondiente a imprescindible; ¿recomiendas este tipo de evaluación para el futuro?, donde únicamente existían dos posibilidades de respuesta «si-no») y cinco preguntas abiertas (¿qué ha supuesto para ti la evaluación en grupo?, ¿consideras que tus preguntas pueden influir en la evaluación del grupo?, ¿qué te ha parecido evaluar a otros compañeros?, ¿qué te ha parecido ser evaluado por otros compañeros?, y ¿por qué recomiendas este tipo de evaluación para el futuro?).

\section{Procedimiento y diseño de la investigación}

El estudio se desarrolló durante los meses de octubre de 2008 a mayo de 2009, en un único grupo de intervención en las asignaturas de actividad física 
para la salud, enseñanza de la actividad física y del deporte, entrenamiento deportivo, y deportes y recreación. En todas ellas se realizaron diferentes actividades (prácticas o teórico-prácticas) en las que se cedió la responsabilidad a los estudiantes en la evaluación de sus compañeros y en su auto-evaluación.

Todas las variables fueron medidas antes y después de la intervención. Una semana previa al inicio del programa, todos los estudiantes fueron informados de que formarían parte de un estudio que «buscaría un nuevo camino de evaluar la enseñanza en la actividad físico-deportiva», y completaron los instrumentos de medida. La duración para completar los cuestionarios fue de unos 15 minutos aproximadamente. Los estudiantes fueron informados de la importancia de su honestidad en la contestación y de que sus respuestas serían confidenciales. Al final de los ocho meses de intervención, todos los estudiantes completaron de nuevo los mismos instrumentos, además de las preguntas sobre la participación en la evaluación.

Programa de cesión de responsabilidad en la evaluación. Se intervino en un entorno ecológico de enseñanza universitaria, planteando diversas actividades en el aula a lo largo de los ocho meses: auto-evaluaciones de situaciones prácticas, evaluación a otros compañeros, toma de decisiones en equipo sobre trabajos presentados por otros compañeros, etc. Todos los estudiantes tuvieron la posibilidad de realizar las mismas actividades programadas. Se dividió cada asignatura en tres momentos diferenciados: una primera fase de experiencia dirigida por el docente, basada en la reproducción del conocimiento, y caracterizada por un sistema de organización masivo-consecutiva, donde las tareas se explicaban al conjunto de la clase y todos los estudiantes las realizaban al mismo tiempo. A mitad de las sesiones que conformaban el programa comenzaba una segunda fase donde se cedía responsabilidad al alumnado en la evaluación de las tareas, caracterizada por un sistema de organización donde la clase se dividía en varios subgrupos, de unos cinco participantes cada uno de ellos, en promedio. En la tercera fase se pedía al alumnado su colaboración en la evaluación del trabajo realizado. Para la participación en la evaluación se convocaban a los grupos donde uno de ellos exponía un trabajo relacionado con la materia y otro grupo lo evaluaba junto al docente. Dicha valoración formaba parte de la calificación final. El docente adoptaba un papel de guía después de la primera fase, utilizando estrategias en la cesión de responsabilidad encaminadas a orientar la enseñanza al desarrollo de la habilidad en la tarea, al interés por la construcción de las actividades y a la valoración del esfuerzo personal y colectivo en el desarrollo de la habilidad.

Para confirmar que esta intervención estaba teniendo un impacto real se recogieron las percepciones en la concesión de autonomía en el aula usando el subfactor autonomía de la escala de mediadores psicológicos 
(González-Cutre et al., 2007). Se midieron en una escala tipo Likert con un rango de puntuación que oscila de 1 (totalmente en desacuerdo) a 5 (totalmente de acuerdo). Tras la prueba t se comprobó el efecto de la intervención $\left(M_{\text {pre }}=3.62\right.$ y $\left.M_{\text {post }}=3.90, p<.01\right)$.

\section{Análisis de datos}

Se utilizó el a de Cronbach para comprobar la consistencia interna de los distintos factores. Fueron calculadas las correlaciones, medias y desviaciones estándares de todas las variables en las dos tomas. Para valorar los efectos del programa se utilizó una prueba t para muestras relacionadas. Para el análisis del contenido del texto el proceso que se siguió fue de tipo interpretativo, con un proceso de categorización del contenido expresado por los estudiantes, y con niveles de fiabilidad inter-observadores superior al $80 \%$. Las diferencias se consideraron estadísticamente significativas cuando $p<.05$. Para el tratamiento de los datos se usó el paquete estadístico SPSS 18.0.

\section{RESULTADOS}

\section{Efectos de la intervención}

La Tabla 1 muestra los resultados para cada una de las variables dependientes, antes y después de la intervención en el programa. La prueba t para muestras relacionadas reveló que el grupo incrementó significativamente en todas las variables en la toma post $(p<.01)$.

\begin{tabular}{|l|l|l|l|}
\hline \multicolumn{2}{|c|}{} & $M$ & $D T$ \\
\hline \multirow{2}{*}{ Interés } & Pre & $4.77^{*}$ & 1.00 \\
\cline { 2 - 4 } & Post & 5.10 & 1.26 \\
\hline Valoración & Pre & $5.00^{*}$ & 1.05 \\
\cline { 2 - 4 } & Post & 5.22 & 1.23 \\
\hline Motivación intrínseca & Pre & $4.25^{*}$ & 1.11 \\
\cline { 2 - 4 } & Post & 5.23 & 1.22 \\
\hline Valor en la cesión & Pre & $6.31^{* *}$ & 1.71 \\
\cline { 2 - 4 } & Post & 7.43 & 1.60 \\
\hline Rol en la cesión & Pre & $5.10^{* *}$ & 1.78 \\
\cline { 2 - 4 } & Post & 6.46 & 1.74 \\
\hline
\end{tabular}

$* \mathrm{p}<.01 ; * \mathrm{*} p<.001$

Tabla 1. Prueba t para muestras relacionadas $(\mathrm{M} \pm \mathrm{DT})$ de las variables dependientes antes y después de la intervención 


\section{Análisis de contenido}

En un primer momento se realizó una lectura de las respuestas de los estudiantes sin anotaciones, se continuó familiarizándose con el texto trascribiendo a ordenador las respuestas que se contestaron a mano y, a continuación, se fueron describiendo los grandes campos temáticos a partir de la reducción de datos textuales propuesta por Gil Flores (1994). De este modo, el contenido de cada respuesta se fue dividiendo en unidades temáticas que expresaban la misma idea. Las transcripciones se convirtieron en múltiples unidades de secuencias textuales que se agrupaban en torno a una unidad temática. A cada una de estas unidades le fue asignado un código en función de su afinidad. De este modo, los investigadores expresaron sus ideas respecto a la constitución de categorías, utilizando para su creación el contraste entre dos compañeros del grupo de investigación, con el fin de buscar acuerdos sobre la visión particular en la existencia de una categoría. Las categorías estaban en constante modificación, redefiniéndose conforme aparecían otros indicadores que las confirmaban o las desautorizaban. Este proceso de acomodación de las categorías a partir de la recodificación permitió una definición más precisa. La interpretación del análisis del contenido del texto se agrupó en los siguientes apartados: contribución en la calificación final, importancia de la participación en la evaluación e implicaciones futuras.

\section{Contribución en la calificación final}

Tras la finalización de la intervención se les preguntó de forma cerrada a los estudiantes en qué medida su participación en la evaluación influiría en la calificación final (Figura 1), donde el 61\% (88) consideró que mucho, mientras que el 29\% (42) la consideraban imprescindible. Cabe destacar que ningún participante la consideró insignificante.

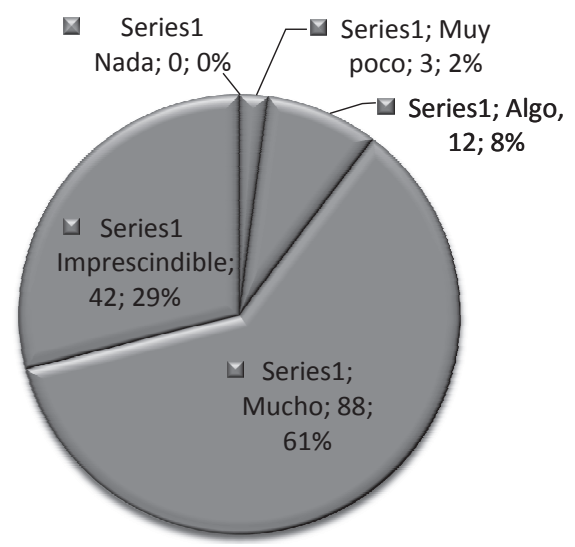

Figura 1. Distribución Porcentual de la Contribución del Estudiante en la Calificación final 
En un intento de profundizar en este orden de importancia, se les preguntó si consideraban que sus preguntas podían influir en la evaluación del grupo (Tabla 2), destacando, en orden de importancia, que dependía del tipo de respuestas que se realizaran, quedando ello reflejado en respuestas como: "Estas preguntas pueden servir para confirmar el buen trabajo del grupo contestando correctamente o certificando por el contrario el no tan buen trabajo del grupo a la hora de realizar su proyecto" (A1). Aunque otro bloque de estudiantes sí que consideraban que se podía influir, bien como apoyo o ayuda a complementar la información, por ejemplo: "Creo que sí que influyen, pero de manera positiva, ya que en principio las preguntas se realizan para que los compañeros puedan explicar mejor algún aspecto» (A61). Por otro lado, el hecho de que se realizaran un determinado tipo de preguntas fue considerado como importante en la influencia en la evaluación, a esta cuestión algunos estudiantes respondieron: «Sí, ya que el grado de dificultad de la pregunta puede poner en apuros al otro grupo» (A32).

\section{Importancia de la participación en la evaluación}

Como queda recogido en la Tabla 2, la evaluación en grupo supuso para el alumnado, principalmente, una aproximación a la realidad laboral, siendo algunos estudiantes sumamente explícitos para dicha cuestión: «Me ha dado la oportunidad de experimentar cómo se lleva a cabo un proyecto que podría ser viable en la realidad» (A37). También, aunque en un orden de importancia mucho menor, fue para ellos una experiencia novedosa, por ejemplo: «Es la primera vez que realizamos una evaluación final de esta forma» (A13), un trabajo en grupo positivo: "Supuso crear un buen ambiente de trabajo y de aportaciones de ideas conjuntas» (A33), lo que les ha llevado a valorarlo como un sistema de evaluación adecuado. Por el contrario, existió un determinado número de estudiantes que lo consideraron un esfuerzo extra y les sirvió para detectar la falta de compañerismo entre el alumnado.

Ante la pregunta de qué les había parecido evaluar a otros compañeros (Tabla 2), la respuesta de una posibilidad de contrastar información, obtener una visión crítica, tomar decisiones y asumir responsabilidades, fue en el recuento de las categorías recogidas la más reiterada en sus respuestas, muestra de ello podría ser: «Ha servido para comparar el estilo y la calidad de los escritos, así como las diferentes formas de interpretar los datos y plasmarlos en un documento» (B4). También lo consideraron como una experiencia positiva para futuras actuaciones profesionales, por ejemplo: «En un futuro no muy lejano nosotros tendremos que evaluar y nos sirve de práctica» (B18) y un trabajo innovador que permite experimentar el rol de evaluador: «Es una modalidad de evaluación de un carácter innovador» (B55). Por el contrario, 
también lo consideraron como problemático por la falta de conocimientos para evaluar y por la dificultad de obtener un consenso grupal: «Debes saber perfectamente la materia que estás evaluando (B67)». Al igual que en la pregunta anterior, el hecho de que se tuviera que dedicar mucho tiempo a dicha evaluación, fue considerado como un trabajo excesivo: "El evaluar los informes de otros compañeros y sus diversos casos lo considero como un trabajo extenso» (B33).

Preocupados por conocer qué les había parecido ser evaluados por otros compañeros (Tabla 2), la respuesta más repetida por el alumnado fue la de que la diversidad de feed-back recibido les permitió obtener una valoración objetiva de los trabajos realizados y, de esa forma, conocer sus propios errores de forma constructiva: "Es una experiencia enriquecedora, ya que te aportan un feed-back sobre el trabajo» (B40). No obstante, otro grupo importante de respuestas coincidieron en considerarlo como negativo, por la falta de conocimiento para la evaluación y la diversidad de criterios en la evaluación por parte del alumnado: «Sientes que no tienes la preparación que tiene el profesor y puede que no se interprete correctamente lo que quieres decir» (B29). Aunque también consideraron que el ser evaluado por compañeros de un mismo nivel era muy positivo: «Me parece bien que se nos dé la oportunidad de ser evaluados por gente cercana a nosotros» (B47). Ante esta diversidad de respuestas, surge la siguiente, que bajo nuestro criterio permitiría regular dicho sistema de participación en la evaluación, y es que por la exigencia del alumnado en la evaluación, ésta debería ser complementada por el docente.

\section{Implicaciones futuras}

El 96\% de los estudiantes recomendó este tipo de evaluación para el futuro, pues lo consideraban como un buen sistema de enseñanza-aprendizaje, novedoso y motivador (Tabla 2): "Pienso que la motivación es mayor» (A26) o "el aprendizaje mediante un trabajo de este tipo es muy significativo» (A40). También destacaban que ofrecía una buena experiencia para el mundo laboral: "Parece resultar útil de cara al futuro» (A18), fomentaba el compañerismo y trabajo en grupo, como por ejemplo: «A la hora de trabajar así se afianzan más las relaciones sociales entre los compañeros y nos da lugar a conocernos más por el tiempo que pasamos juntos» (A48), permitía mejorar la exposición en público, además de servir de control del trabajo realizado. No obstante, otra categoría en la que también insistían era que lo recomendarían siempre y cuando se mejoraran algunos aspectos de organización, como podría ser acortar el número de preguntas y tiempo de exposición, menos dedicación en tiempo, etc. 


\begin{tabular}{|c|c|}
\hline $\begin{array}{l}\text { ¿Consideras que tus preguntas pueden influir en la evaluación } \\
\text { del grupo? }\end{array}$ & Recuento \\
\hline Dependiendo de las respuestas & 44 \\
\hline Sí, como apoyo o ayuda a complementar información & 37 \\
\hline Dependiendo de las preguntas & 20 \\
\hline Sí, detectando errores & 13 \\
\hline No significativamente & 12 \\
\hline Sí, enriqueciendo activamente el proceso de enseñanza-aprendizaje & 11 \\
\hline Sí, más positivo cuantos más evaluadores & 7 \\
\hline Sí, igual de válidas que las de los profesores & 7 \\
\hline ¿Qué ha supuesto para ti la evaluación en grupo? & Recuento \\
\hline Aproximación a la realidad laboral & 84 \\
\hline Experiencia novedosa & 36 \\
\hline Trabajo en grupo positivo & 31 \\
\hline Sistema de evaluación adecuado & 27 \\
\hline Esfuerzo extra & 25 \\
\hline Muestra de falta de compañerismo & 13 \\
\hline Adquisición de conocimientos & 10 \\
\hline ¿Qué te ha parecido evaluar a otros compañeros? & Recuento \\
\hline $\begin{array}{l}\text { Posibilidad de contrastar información, obtener una visión crítica, } \\
\text { tomar decisiones y asumir responsabilidades }\end{array}$ & 86 \\
\hline Positivo como experiencia y para futuras actuaciones profesionales & 55 \\
\hline $\begin{array}{l}\text { Un trabajo innovador que permite experimentar un nuevo rol (eva- } \\
\text { luador) }\end{array}$ & 39 \\
\hline $\begin{array}{l}\text { Problemático, por la falta de conocimientos para evaluar y por la di- } \\
\text { ficultad de obtener un consenso grupal }\end{array}$ & 29 \\
\hline Trabajo excesivo (duradero) & 25 \\
\hline Muestra de carencias de los compañeros & 8 \\
\hline Positivo porque ha sido un proceso muy objetivo (anonimato) & 6 \\
\hline ¿Qué te ha parecido ser evaluado por otros compañeros? & Recuento \\
\hline $\begin{array}{l}\text { Con el feedback múltiple, he podido obtener una valoración objetiva } \\
\text { de los trabajos y conocer mis propios errores de modo constructivo }\end{array}$ & 83 \\
\hline $\begin{array}{l}\text { Negativo por la falta de conocimientos para evaluar y la existencia } \\
\text { de criterios diferentes de evaluación }\end{array}$ & 32 \\
\hline $\begin{array}{l}\text { Positivo por ser evaluado por compañeros con el mismo nivel de co- } \\
\text { nocimientos }\end{array}$ & 20 \\
\hline
\end{tabular}




\begin{tabular}{|l|c|} 
Por la exigencia de los compañeros la evaluación deber ser comple- & 16 \\
tada por el docente & 8 \\
Adquisición de experiencias para el futuro (ser evaluado) & 7 \\
Ha hecho que me esfuerce más (motivación) & 5 \\
Muestra de falta de objetividad del alumnado & Recuento \\
\hline ¿Por qué recomiendas este tipo de evaluación para el futuro? & 36 \\
Buen sistema de enseñanza-aprendizaje, novedoso y motivador & 27 \\
Ofrece experiencia para el mundo laboral & 15 \\
Fomenta el compañerismo y trabajo en grupo & 15 \\
Mejorando aspectos de organización (Acotando el número de pre- \\
guntas y tiempo de exposición, con menos dedicación, adelanto de la \\
fecha de exposición, aumento en el tiempo de exposición, etc.) \\
Mejora la exposición en público & 14 \\
Permite llevar un control de lo trabajado & 13 \\
Complementando con un examen teórico & 5 \\
Dependiendo de la asignatura & 5 \\
\hline
\end{tabular}

Tabla 2. Distribución del recuento de las categorías obtenidas ante las preguntas

\section{DISCUSIÓN Y CONCLUSIONES}

El presente estudio se centró en conocer la influencia que un programa de trabajo de cesión de responsabilidad en la evaluación tenía en la creencia del alumnado sobre dicho programa y en la mejora de la percepción de autonomía, la motivación intrínseca, y el valor y rol de la evaluación. Tras el análisis de resultados se pudo aceptar la hipótesis de partida. Al respecto, numerosos estudios e investigaciones (Álvarez Méndez, 2007; Vera y Moreno, 2008) vienen profundizando en la necesidad de proponer estrategias diferentes en la evaluación del alumnado, argumentando, fundamentalmente, la utilización de una evaluación que puede incorporarse al proceso formativo desde el protagonismo del discente, abandonando las características de un modelo tradicional basado en la medición del rendimiento y facilitando oportunidades para valorar la comprensión de los contenidos y el esfuerzo empleado para su consecución. Esta circunstancia guarda cierto paralelismo con aquellas investigaciones (Moreno, Vera, y Del Villar, 2010; Prusak, Treasure, Darst y Pangrazi, 2004; Wallhead y Ntoumanis, 2004; Ward, 2006) que han subrayado la importancia de dirigir la enseñanza de la educación física y del deporte hacia un incremento de la autonomía, centrada en la toma de decisiones del alumnado, donde la cesión de responsabilidad ocupa un lugar destacado en el desarrollo de la autonomía responsable. 
Así pues, la autonomía juega un papel importante en el impacto que tiene sobre los factores situacionales que afectan a la motivación. Al respecto, algunas investigaciones (Moreno, Gómez y Cervelló, 2010; Vallerand y Rousseau, 2001) han informado de niveles más altos de motivación intrínseca en aquellos estudiantes a los que se les permitía elegir las tareas en el aula de educación física. Los datos obtenidos en nuestro estudio, nos permiten apoyar este tipo de observaciones también en el nivel universitario. Esta participación construida desde la autonomía de acción resulta necesaria si el aumento en la valoración de las clases se ha visto mejorado cuando el docente ha generado un clima motivacional que proporciona autonomía y responsabilidad al alumnado (Cervelló, Jiménez, Del Villar, Ramos y SantosRosa, 2004; Moreno et al, 2006). De este modo, las oportunidades de los estudiantes para participar en la evaluación han presentado efectos positivos en la motivación intrínseca cuando se han planteado desde estilos de enseñanza que suministran a los estudiantes la oportunidad de elegir y de tomar decisiones (Hassandra, Goudas y Chroni, 2003). Parece que la motivación es un factor que nos ayuda a comprender las diferentes percepciones individuales que tienen lugar en los estudiantes. De este modo, $\mathrm{Li}$, Lee y Solmon (2005) indican que aquellos estudiantes que están más motivados se perciben más competentes.

El resultado del análisis del contenido del texto confirmó la importancia concedida por el alumnado a la participación en la evaluación de forma significativa. Además, en el caso de la intervención donde cada grupo tenía la oportunidad de repartir la calificación obtenida de forma dispar, este tipo de intervención contribuye a que afloren posibles conflictos dentro del grupo, a tenor del nivel de implicación de cada miembro en el desarrollo del trabajo. En este sentido, la construcción de las categorías de análisis pueden constituir temas que afronten la didáctica a partir de ofrecer al profesorado la posibilidad de plantear un debate con implicaciones prácticas y reflexivas acerca de cómo desarrollar estrategias de cesión de responsabilidad ante la evaluación del alumnado.

A tenor de los resultados obtenidos, consideramos que la cesión de responsabilidad al alumnado en el proceso de evaluación de parte de los productos de enseñanza-aprendizaje ha sido positiva. Por un lado, la preparación de un trabajo en grupo, para su posterior defensa, permite que el estudiante calibre el papel que desempeña dentro del grupo, haciendo palpable el hecho que de su implicación y rendimiento se verá afectada la evaluación, u posterior calificación, tanto suya como de sus compañeros. Además, la formulación de preguntas a otros grupos hace que el estudiante adopte un rol de evaluador, similar al que podría desempeñar en futuras tareas como egresado universitario o profesional. 
Estas situaciones de evaluación pueden ser de gran utilidad, no solo en el ámbito de la enseñanza universitaria, sino también en el de la educación física secundaria o la formación profesional (e.g. titulaciones profesionales o títulos federativos), así como otros niveles formativos como los de post-grado o master. No obstante, creemos que se hace preciso de un buen conocimiento de la dinámica de evaluación, del alcance de las decisiones adoptadas, y la repercusión que ello puede tener en una calificación ulterior. Se hace preciso crear un clima de aula adecuado, exento de situaciones donde el estudiante pueda utilizar esta responsabilidad para hacer "daño» a otros compañeros, es decir, no utilice su rol de forma constructiva. Así, también creemos que este tipo de estrategias evaluativas son de interés cuando el alumnado conoce el contenido que está evaluando, y por supuesto, el que está exponiendo. La calidad de sus intervenciones se torna en un indicativo que puede mostrar la claridad de los conceptos allí expuestos.

No obstante, creemos que este estudio presenta algunas limitaciones. Por ejemplo, la formación de los grupos se permitió que fuera a propuesta de los propios estudiantes, lo cual limita en cierto modo valorar la capacidad del mismo de acoplarse a diferentes dinámicas de trabajo. El resultado de ello, es que es posible que el alumnado "pacte» el nivel de implicación en el trabajo, y el reparto de la calificación posterior sea equitativo si se da una situación de mayoría, evitando así conflictos entre compañeros cuya percepción del esfuerzo sea dispar al de otros miembros del grupo. Por ello, recomendamos hacer futuras intervenciones con una formación de grupos azarosa, para lo cual se podría aplicar en grupos de cursos inferiores, donde las relaciones sociales no están tan arraigadas todavía. 


\section{REFERENCIAS BIBLIOGRÁFICAS}

Álvarez Méndez, J.M. (2007). La evaluación formativa. Cuadernos de Pedagogía, 364, 96-100.

Aracil, A. (2010). A new conception of knowledge, a new conception of learning: from Tetris to Rubick's cube. Medical Education, 44 (2), 215.

Callaghan, D., Watts, W.E., McCullough, D.L., Moreau, J. T., Little, M A., Gamroth, L.M. y Durnford, K.L. (2009). The experience of two practice education models: Collaborative learning unit and preceptorship. Nurse Education in Practice, 9 (4), 244-252.

Cervelló, E., Jiménez, R., Del Villar, F., Ramos, L. y Santos-Rosa, F. J. (2004). Goal orientations, motivational climate, equality, and discipline of Spanish physical education students. Perceptual and Motor Skills, 99, 271-283.

Conde, C. Sáenz-López, Carmona, J., González-Cutre, D., Martínez Galindo, C., y Moreno, J. A. (2010). Validación del Cuestionario de Percepción de Soporte de la Autonomía en el Proceso de Entrenamiento (ASCQ) en jóvenes deportistas españoles. Estudios de Psicología, 31 (2), 145-157.

Conroy, D. y Coatsworth, J. (2007). Assessing autonomy-supportive coaching strategies in youth sport. Psychology of Sport and Exercise, 8, 671-684.

Declaración de Bolonia (1999). Declaración conjunta de los Ministros Europeos de Educación. Bolonia. Recuperado de: www.universia.es/contenidos/universidades/documentos/ Universidades-documbolonia.htm

Declaración de Praga (2001). Hacia el Espacio Europeo de Educación Superior. Comunicado de la Conferencias de Ministros Europeos responsables de
Educación Superior. Recuperado de: www.crue.org/comcumbrepraga.htm

Del Valle, S., Ruiz, L.M., Velázquez, R. y Díaz, P. (2005). ¿Sabemos qué contenidos aprenden los escolares en educación física? European Journal of Human Movement, 13, 87-106.

Del Villar, F. (Ed.) (2006). Libro Blanco del título de grado en Ciencias de la Actividad Física y del Deporte. ANECA. Recuperado de: http://www.aneca.es/ activin/activin_conver_LLBB.asp

Elliott, N. y Higgins, A. (2005). Self and peer assessment: does it make a difference to student group work? Nurse Education in Practice, 5 (1), 40-48

Gil Flores, J. (1994). Análisis de datos cualitativos. Aplicaciones a la investigación educativa. Barcelona: PPU.

González-Cutre, D., Martínez Galindo, C., Alonso, N., Cervelló, E., Conte, L. y Moreno, J.A. (2007). Las creencias implícitas de habilidad y los mediadores psicológicos como variables predictoras de la motivación autodeterminada en deportistas adolescentes. En J. Castellano, y O. Usabiaga (Eds.), Investigación en la Actividad Física y el Deporte II (pp. 407-417). Vitoria: Universidad del País Vasco.

Goudas, M., Biddle, S.J.H. y Fox, K. (1994). Achievement goal orientations and intrinsic motivation in physical fitness testing with children. Pediatric Exercise Science, 6, 159-167.

Hassandra, M., Goudas, M. y Chroni, S. (2003). Examining factors associated with intrinsic motivation in physical education: a qualitative approach. Psychology of Sport and Exercise, 4, 211-223.

Li, W., Lee, A. M. y Solmon, M. A. (2005). Relationships among dispositional 
ability conceptions, intrinsic motivation, perceived competence, experience, persistence, and performance. Journal of Teaching in Physical Education, 24, 51-65.

Mateo, J. y Martínez, F. (2005). La evaluación alternativa de los aprendizajes. Barcelona: ICE de la Universidad de Barcelona.

Moreno, J. A., Gómez, A. y Cervelló, E. (2010). Un estudio del efecto de la cesión de autonomía en la motivación sobre las clases de educación física. Motricidad. European Journal of Human Movement, 24, 1-21.

Moreno, J.A., González-Cutre, D. y Chillón, M. (2009). Preliminary validation in Spanish of a scale designed to measure motivation in physical education classes: the Perceived Locus of Causality (PLOC) Scale. The Spanish Journal of Psychology, 12 (1), 327-337.

Moreno, J.A. y Vera, J.A. (2008). Un estudio experimental de las diferencias por género en la percepción de competencia a partir de la cesión de responsabilidad en las clases de educación física. Revista Iberoamericana de Educación, 46, 1-10.

Moreno, J.A., Vera, J.A. y Cervelló, E. (2006). Evaluación participativa y responsabilidad en educación física. Revista de Educación, 340, 731-754.

Moreno, J.A., Vera, J.A. y Cervelló, E. (2009). Efectos de la cesión de responsabilidad de la evaluación en la motivación y la competencia percibida en el aula de educación física. Revista de Educación, 348, 423-440.

Moreno, J.A., Vera, J.A. y Del Villar, F. (2010). Search for autonomy in motor task learning in physical education university students. European Journal of Psychology of Education, 25 (1), 37-47.
Özgüngör, S. (2009). The relationships between students' evaluations of teaching behaviors and self-efficacy beliefs. Procedia-Social and Behavioral Sciences, 1(1), 2687-2691.

Prusak, K.A., Treasure, D.C., Darst, P.W. y Pangrazi, R.P. (2004). The effects of choice on the motivation of adolescent girls in physical education. Journal of Teaching in Physical Education, 23, 19-29.

Vallerand, R.J. y Rousseau, F.L. (2001). Intrinsic and extrinsic motivation in sport and exercise: A review using the hierarchical model of intrinsic and extrinsic motivation. En R. N. Singer, H. A. Hausenblas y C. M. Janelle (Eds.), Handbook of Sport Psychology (2 ${ }^{\mathrm{a}}$ ed., pp. 389-416). New York: John Wiley \& Sons.

van Genni, N.A.E., Segers, M.S.R. y Tillema, H.H. (2009). Peer assessment for learning from a social perspective: The influence of interpersonal variables and structural features. Educational Research Review, 4(1), 41-54

van Genni, N.A.E., Segers, M.S.R. y Tillema, H.H. (2010). Peer assessment as a collaborative learning activity: The role of interpersonal variables and conceptions. Learning and Instruction, 20(4), 280-290

Vera, J.A. y Moreno, J.A. (2008). La enseñanza de la responsabilidad en el aula de educación física escolar. Habilidad Motriz, 32, 39-43.

Wallhead, T.L. y Ntoumanis, N. (2004). Effects of a sport education intervention on students' motivational responses in physical education. Journal of Teaching in Physical Education, 23, 4-18.

Ward, P. (2006). What we teach is as important as how we teach it. Journal of Physical Education, Recreation \& Dance, 77, 20-23. 


\section{PALABRAS CLAVE}

Autonomía; toma de decisiones; evaluación; educación física; educación superior; innovación docente.

\section{KEYWORDS}

Autonomy; decision-making; evaluation; physical education; higher education; teaching innovation.

\section{PERFIL ACADÉMICO Y PROFESIONAL DE LOS AUTORES}

Juan Antonio Moreno Murcia, Profesor en la Facultad de Ciencias Sociosanitarias de la Universidad Miguel Hernández de Elche. Docente de la asignatura «Enseñanza de la actividad física y del deporte», así mismo, también es investigador del Grupo de Investigación en Comportamiento Motor y sus líneas de investigación se centran en el estudio de los procesos de enseñanza-aprendizaje de las actividades físico-deportivas.

Adolfo Aracil Marco, Profesor en la Facultad de Ciencias Sociosanitarias de la Universidad Miguel Hernández de Elche. Docente de la asignatura «Bases biológicas del cuerpo humano», y miembro del Grupo de Investigación en Acondicionamiento Físico Saludable. Sus líneas de investigación se centran en el estudio de las variables biológicas del comportamiento humano en las actividades físico-deportivas.

Raúl Reina Vaillo, Profesor en la Facultad de Ciencias Sociosanitarias de la Universidad Miguel Hernández de Elche. Especialista en actividades físico-deportivas para personas con discapacidad. Miembro del Grupo de Investigación en Aprendizaje y Control Motor y su línea de investigación se centra en el estudio de la actividad física adaptada.

Dirección de los autores: Juan Antonio Moreno Murcia Universidad Miguel Hernández de Elche Centro de Investigación del Deporte Avenida de la Universidad, s/n. 03202 Elche (Alicante)

E-mail: j.moreno@umh.es fito@umh.es rreina@umh.es

Fecha Recepción del Artículo: 11. Octubre. 2011 Fecha Revisión del Artículo: 24. Mayo. 2012 Fecha de Aceptación para publicación: 21. Junio. 2012 Fecha Revisión para publicación: 24. Junio. 2013 
